\title{
Relações Cineantropométricas e Fisiológicas Durante Exercício Incremental em Esteira Rolante
}

\author{
Kineanthropometric and Physiological Relations During \\ Incremental Treadmill Exercise
}

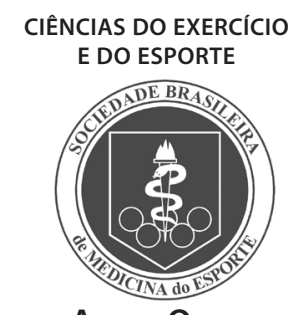

Artigo Original

\author{
Anderson Souza-Castelo Oliveira ${ }^{1,2}$ \\ Ramires Alsamir Tibana' \\ Fernando de Aguiar' \\ Edilberto Barros ${ }^{3}$ \\ Priscila de Brito Silva ${ }^{3}$ \\ 1. Centro Universitário Unieuro, \\ Brasília, DF. \\ 2. Aalborg University, Aalborg, \\ Dinamarca. \\ 3. Universidade Católica de Brasília, \\ Brasilia, DF.
}

Endereço para correspondência: Center for Sensory-Motor Interaction

Department of Health Science and technology, Aalborg University

Fredrik Bajers Vej 7, DK 9220.

Aalborg - Denmark

E-mail: oliveira@hst.aau.dk

\begin{abstract}
RESUMO
Testes incrementais em esteira rolante são muito utilizados para prescrição de treinamentos, havendo alterações fisiológicas e cinemáticas devido à natureza de incremento da intensidade do exercício. Contudo, a antropometria é um fator que não apresenta consenso na literatura. Neste sentido, os objetivos do presente estudo foram verificar o efeito de exercício incremental na concentração de lactato sanguíneo ([LAC]), e glicose ([GLI]), como também na frequência (FP) e amplitude de passadas (AP). Além disso, correlacionar valores de dados antropométricos (massa, altura, \% de gordura) com as alterações existentes das [LAC] e [GLI]. Treze voluntários saudáveis realizaram teste incremental em esteira rolante (início a $8 \mathrm{~km} / \mathrm{h}$, com incrementos de $1 \mathrm{~km} / \mathrm{h}$ a cada $3 \mathrm{~min}$ até a exaustão). Antes e 3 min após a realização do teste incremental foram registrados os valores de [LAC] e [GLI]. Durante cada intensidade do teste incremental foram realizadas avaliações da AP e FP por meio de filmagens no plano sagital. Como resultados, verificou-se que a [LAC] e [GLI] antes do teste incremental $(1,94 \pm 0,4 \mathrm{mmol} / \mathrm{L}$ e $94,3 \pm 7 \mathrm{mg} / \mathrm{dL}$, respectivamente) foram significantemente menores que os valores encontrados após o teste incremental $(9,51 \pm 2,7 \mathrm{mmol} / \mathrm{L}$ e $126 \pm 16 \mathrm{mg} /$ $\mathrm{dL}$, respectivamente). Além disso, verificou-se aumento significante e gradativo da AP e FP ao longo do teste incremental. Correlações inversas e significantes $(p<0,05)$ foram encontradas entre a [LAC] e altura, massa corporal e o nível de treinamento dos voluntários. Conclui-se que a execução do teste incremental altera parâmetros cinemáticos (pela necessidade de deslocamento mais rápido) e fisiológicos (processo de fadiga muscular, em decorrência do aumento da velocidade). Além disso, voluntários menores e mais leves podem apresentar maiores concentrações de metabólitos.
\end{abstract}

Palavras-chave: fadiga muscular, corrida, teste incremental, cinemática.

\begin{abstract}
Incremental treadmill tests are widely used for prescription of aerobic and anaerobic training. Physiological and kinematics parameters are changed by the progressive nature of this activity. However, there is no consensus on how anthropometric characteristics may affect the incremental test performance. Therefore, the aims of this study were (1) to verify the effects of the incremental treadmill test on the blood lactate concentration ([LAC]), glucose concentration ([GLU]), step frequency (SF) and step length (SL), and (2) to correlate anthropometric data (body mass, height, \%body fat) to changes in [LAC] and [GLU]. Thirteen healthy males volunteered to this study and performed an incremental running test on a treadmill (start: $8 \mathrm{~km} / \mathrm{h}$, with progressive increases [1 km/h] at each three minute until exhaustion). [LAC] and [GLU] were registered before and $3 \mathrm{~min}$ after the incremental test performance. SF and SL were registered for each running speed by digital images at the sagital plane. As results, we verified that [LAC] and [GLU] before the incremental test $(1.94 \pm 0.4 \mathrm{mmol} / \mathrm{L}$ and $94.3 \pm 7 \mathrm{mg} / \mathrm{dL}$, respectively) were significantly lower $(p<$ $0.05)$ than after the incremental test $(9.51 \pm 2.7 \mathrm{mmol} / \mathrm{L}$ and $126 \pm 16 \mathrm{mg} / \mathrm{dL}$, respectively). In addition, SF and SL presented significant increase $(p<0.05)$ throughout the incremental test. [LAC] presented inverse and significant correlations $(p<0.05)$ to height and body mass. Thus, an incremental treadmill running test affects kinematic (SF and SL) and physiological conditions (muscular fatigue, as consequence of the speed increase). Besides this, shorter and lighter subjects may present higher metabolite concentrations after this type of test.
\end{abstract}

Keywords: muscular fatigue, running, incremental test, kinematics. 


\section{INTRODUÇÃO}

A determinação de índices de treinamento aeróbio e anaeróbio pode ser realizada por meio de exercícios incrementais, uma vez que o aumento progressivo da intensidade de exercício oferece o monitoramento das alterações fisiológicas, as quais podem refletir a capacidade aeróbia do indivíduo ${ }^{(1,2)}$. Diversos estudos relatam a utilização de índices como o limiar aeróbio, limiar anaeróbio e limiar ventilatório, os quais são amplamente utilizados para determinar intensidades para a aplicação de protocolos de treinamento ${ }^{(1,3)}$. Durante um teste incremental, há aumento da concentração de glicose saguínea ([GLI] ${ }^{(4)}$, juntamente com o acúmulo de metabólitos (íons $\mathrm{H}^{+}$, entre outros). Entre estes, a concentração de lactato sanguíneo ([LAC]) é amplamente utilizada para determinar a demanda metabólica ao longo do teste ${ }^{(5)}$. Parâmetros ventilatórios também podem ser avaliados para determinar esse nível de aumento no esforço, como o aumento da ventilação (VE), a qual apresenta relação com a intensidade em que o limiar anaeróbio (LAn) é determinado pela [LAC] ${ }^{(6)}$.

Análises biomecânicas em exercícios de corrida utilizam principalmente a frequência de passadas (FP) e amplitude de passadas (AP) ${ }^{(7)}$. A escolha ideal de FP e AP podem ser influenciadas por fatores como a superfície de contato e fadiga muscular ${ }^{\left({ }^{8}\right)}$. Foi demonstrado que pode haver aumento concomitante da FP e AP durante o exercício incremental, o que possui relação com a necessidade de deslocamento mais rápido durante o teste, a medida que a velocidade aumenta $a^{(9,10)}$. Outros estudos sobre cinemática de corrida relacionam o tempo de contato com o solo (T-cont) ao longo de diferentes velocidades, verificando que quanto maior a velocidade, menor pode ser este tempo de contato(6). Alterações de parâmetros cinemáticos são importante evidência de que existe aumento no esforço realizado, e a relação destas variáveis com alterações fisiológicas também tem sido de grande valia para melhor prescrição e entendimento de testes incrementais ${ }^{(3)}$, alterações na [LAC] e consumo de $\mathrm{O}_{2}$ durante velocidade constante são acompanhados de alterações cinemáticas, como o aumento da FP(9,11).

Embora já existam estudos relacionados com as alterações biomecânicas e fisiológicas durante testes incrementais, existem ainda aspectos importantes que não foram investigados. Uma vez que o teste deve ser padronizado para pessoas de mesmo condicionamento, verificamos que quanto maior o nível de treinamento dos indivíduos, mais altas podem ser as velocidades iniciais dos testes ${ }^{(3)}$. No entanto, parâmetros mecânicos podem ser alterados devido ao aumento da velocidade, e também relações antropométricas podem acarretar alterações frente a estas intensidades.

Estudos anteriores que investigaram influências de parâmetros antropométricos na corrida demonstraram que, durante a corrida, a utilização da frequência e amplitude de passadas preferidas pode ser fator que aumenta a economia de corrida(3). No entanto, Cavanagh e $\mathrm{Kram}^{(8)}$ não evidenciaram relacionamento entre variáveis antropométricas e parâmetros cinemáticos como a AP e FP. Além disso, estes autores apresentaram ausência de alterações nos parâmetros cinemáticos quando a massa dos indivíduos foi alterada (acréscimos de 1,1 kg em cada tornozelo). Espera-se que exista influência de parâmetros antropométricos em variáveis cinemáticas durante a corrida, mas ainda não existe consenso sobre este assunto.

Neste sentido, o objetivo do presente estudo foi verificar o efeito da execução de um exercício incremental em esteira rolante nas [GLI] e [LAC], bem como na AP e FP durante este teste. Além disso, houve o objetivo de correlacionar as alterações existentes nas concentrações destes substratos com parâmetros antropométricos (massa, altura, \% de gordura, comprimento de coxa e perna). Como hipóteses, o presente estudo espera que existam aumentos nas [GLI] e [LAC], em parâmetros cinemáticos (FP e AP) com o desenvolvimento do teste incremental, como também que aspectos como maiores massas, alturas e \% de gordura causem aumentos nas concentrações de metabólitos, principalmente da [LAC].

\section{MÉTODOS}

\section{Voluntários}

Participaram deste estudo 13 homens saudáveis com média de idade de 29,9 $\pm 6,3$ anos, altura de 177,8 $\pm 5,5 \mathrm{~cm}$, massa corporal de $77,2 \pm 4 \mathrm{~kg}$, percentual de gordura corporal (\%gord) $12,5 \pm 5 \%$. Esses voluntários possuíam prática de exercícios de corrida, embora sem treinamento sistematizado na categoria. Os sujeitos não possuíam histórico de lesões musculoesqueléticas nos membros e coluna vertebral. O protocolo deste estudo foi aprovado pelo Comitê de Ética em Pesquisa do Centro Universitário Unieuro (parecer: 019/2009, protocolo: 014/09) e os voluntários assinaram Termo de Consentimento Livre e Esclarecido antes de realizarem os testes.

\section{Delineamento experimental}

Os voluntários realizaram os testes em apenas uma visita. Inicialmente foram explicados os procedimentos experimentais, e em seguida foi feita a análise de composição corporal dos indivíduos. Após a composição corporal, foi realizado teste incremental em esteira rolante, até a exaustão voluntária dos sujeitos. Ao longo dos testes, foram registrados valores de AP e FP, além de valores referentes a frequência cardíaca (FC) e ventilação (utilizada para o cálculo do LAn). Antes e após a realização do teste incremental foram realizadas coletas de sangue para análises de [GLI] e [LAC]. Todos os testes foram realizados no período vespertino ou noturno (entre 15:00 e 19:00). Os voluntários foram instruídos a não realizarem refeições no período de duas horas anteriores ao teste, como também foram instruídos a não ingerirem bebidas alcoólicas, tampouco bebidas que contém estimulantes (cafeína, efedrina entre outros).

\section{Antropometria}

Para análise do crescimento físico, a massa corporal dos sujeitos foi obtida em balança digital, da marca Filizola ${ }^{\oplus}$, com precisão de 0, 1 kg, e a estatura foi determinada em estadiômetro de madeira, com precisão de $0,1 \mathrm{~cm}$. A composição corporal foi determinada pela avaliação de sete dobras (abdominal, tricipital, suprailíaca, peitoral, axilar média, subescapular e coxa) seguindo o protocolo de Jackson e Pollock ${ }^{(12)}$. Três medidas foram obtidas para cada ponto anatômico no hemicorpo direito, por um único avaliador, por meio de um adipômetro com precisão de 0,1 cm (Sanny, AD-1010, São Paulo, Brasil). Os valores das dobras foram inseridos no software Galileu (Micromed, Galileu, Brasília, Brasil) para o cálculo da composição corporal e do percentual de gordura.

\section{Determinação do limiar anaeróbio (LAn)}

Todos os voluntários realizaram teste incremental em esteira rolante (Micromed, Centurion, Brasília, Brasil) para a determinação do LAn. A velocidade inicial do teste foi de $8 \mathrm{~km} / \mathrm{h}$ com inclinação de $1 \%$, houve incremento de $1 \mathrm{~km} / \mathrm{h}$ a cada três minutos de teste até $16 \mathrm{~km} / \mathrm{h}$ (a partir desta velocidade houve incremento de $1 \%$ na inclinação da esteira a cada minuto). Durante a execução do teste, valores de FC e VE foram registrados por meio de software específico (Micromed, FlowMet, Brasília, Brasil). Para a determinação do LAn, verificou-se a segunda inflexão da curva da $V E$, que possui validade comprovada para indicação deste índice aeróbio ${ }^{(1)}$. Todas as inflexões foram determinadas por um mesmo avaliador, com experiência na determinação deste índice.

\section{Determinação da frequência e amplitude de passadas}

As variáveis cinemáticas foram registradas com câmera de vídeo (Sony, DCR-TRV351, Tóquio, Japão) com frequência de amostragem de $30 \mathrm{~Hz}$, colocada a $6 \mathrm{~m}$ de distância do plano sagital da esteira. Tais vídeos foram desentrelaçados por meio do software VirtualDub e a frequência utilizada para as análises foi de $60 \mathrm{~Hz}$. Todas as velocidades dos testes foram filmadas, e as imagens dos 30 segundos finais de cada estágio foram armazenadas em microcomputador para posteriores análises. 
Os cálculos da amplitude de passada (distância entre dois toques do mesmo pé no solo) e frequência de passadas (número de passadas realizadas em um minuto) foram realizados por meio da utilização de softwares específicos como o VirtualDub, Motion Analysis Tools e Microsoft Excel para cada velocidade do teste.

\section{Determinação das variáveis metabólicas (glicose e lactato sanguíneo)}

Anteriormente ao protocolo incremental, os voluntários permaneceram em repouso por cinco minutos na posição sentada. Ao final deste tempo foram coletadas amostras de sangue para avaliação da [GLI] (Roche, Accu-Check Advantage, Brasil) e da [LAC] (Roche AccuCheck, Performa, Brasil). Três minutos após o término do protocolo incremental, foram realizadas novas medições da [GLI] e [LAC]. Para análises sanguíneas utilizaram-se gazes e álcool 70\% foi feita a assepsia do dedo utilizado na coleta, e por meio de punção em sua polpa lateral, utilizando lancetador e luvas descartáveis, coletando uma gota de sangue que foi colocada no centro da zona de teste da fita reativa para análise das concentrações de lactato e glicose sanguínea. Tanto as amostras referentes ao repouso, quanto às referentes ao final do teste incremental, foram analisadas logo após sua coleta.

\section{Análises estatísticas}

Todos os resultados foram expressos em valores médios e desvio padrão ( $\pm D P)$. Comparações entre os valores de [GLI] e [LAC] antes e após o teste incremental foram realizadas por meio do teste $t$ de Student para amostras dependentes. Foi realizado o cálculo da diferença existente entre os valores de [GLI] e [LAC] após e antes do teste incremental (delta-GLI e delta-LAC). Os valores de delta-GLI e delta-LAC foram correlacionados com valores de massa, altura, porcentagem de gordura corporal (\%gord), nível de treinamento (N-TR) e número de estágios realizados (EST). Estas correlações foram feitas por meio do teste de correlação de Pearson. O efeito da velocidade do teste incremental nos valores de AP e FP foi realizado por meio de análise de variância (one-way Anova), complementado pelo teste post hoc de Tukey. Além disso, foi calculada a variação da AP e FP entre cada incremento de carga (ex: entre $8 \mathrm{~km} / \mathrm{h}$ e $9 \mathrm{~km} / \mathrm{h}$ ), sendo esta uma porcentagem de alteração entre os estágios. $\mathrm{O}$ efeito da velocidade do teste incremental nos valores de porcentagem de alteração da AP e FP foi realizado por meio de análise de variância (Anova), complementado pelo teste post hoc de Tukey. Foram realizadas também correlações entre esta porcentagem de alteração da AP e FP e os valores de massa, altura, comprimento da coxa e comprimento da perna por meio de correlações de Pearson. Para todas as análises, o nível de significância foi estabelecido em $p \leq 0,05$.

\section{RESULTADOS}

Os resultados de desempenho durante o teste incremental são exibidos na tabela 1. O tempo médio de exaustão para os voluntários foi de 26,4 1,2 minutos (mínimo = 24 minutos, máximo 27 minutos).

Tabela 1. Valores médios ( $\pm D P$ ) de limiar anaeróbio (LAn), frequência cardíaca no LAn (FC-LAn), velocidade máxima alcançada no teste incremental (vMAX) e percentual da velocidade máxima que a velocidade do LAn representa (\%vMAX) $(n=13)$.

\begin{tabular}{c|c|c|c}
\hline LAn $(\mathbf{k m} / \mathbf{h})$ & FC-LAn (bpm) & $\operatorname{vMAX}(\mathbf{k m} / \mathbf{h})$ & \%vMAX (\%) \\
\hline $11,1 \pm 1,4$ & $165 \pm 9$ & $15,1 \pm 1,2$ & $73,4 \pm 6$ \\
\hline
\end{tabular}

Os valores de [GLI] e [LAC] antes do teste incremental foram significantemente menores que os valores encontrados após o teste incremental ( $p<0,001)$ (gráfico 1). Amostras de sangue de dois voluntários podem ter sido contaminadas e, portanto, foram desconsideradas, totalizando $n=11$.

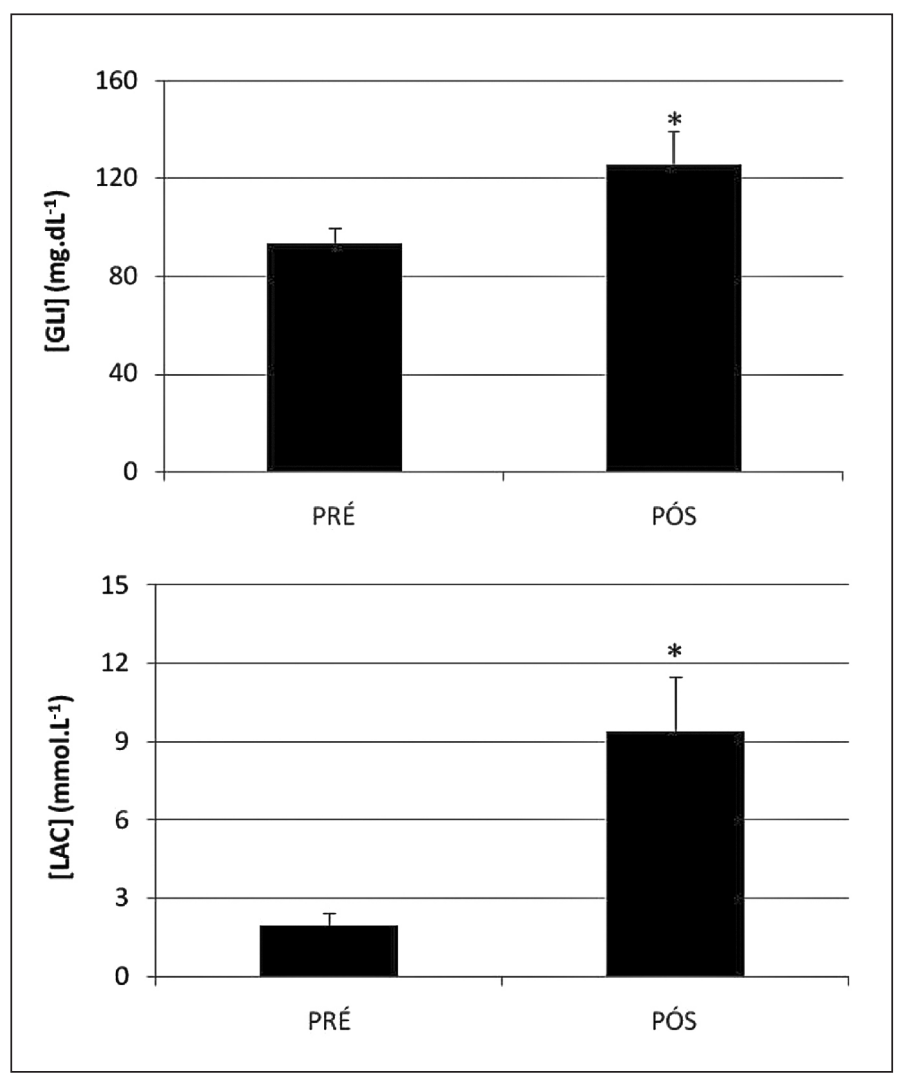

Gráfico 1. Valores médios ( $\pm D P$ ) de concentração da glicose sanguínea ([GLI], gráfico A) e de lactato sanguíneo ([LAC], gráfico B) antes (PRÉ) e após (PÓS) o teste incremental em esteira rolante $(n=11)$. ${ }^{*}$ Denota diferença significante em relação à PRÉ, por meio do teste $t$ de Student $(p<0,05)$.

Os valores de AP e FP apresentaram efeito da velocidade por meio do teste de Anova ( $<0,0001)$. Para a AP (gráfico 2), o teste post hoc apresentou valores significantemente menores das velocidades iniciais $(8,9,10$ e $11 \mathrm{~km} / \mathrm{h})$ em relação às velocidades finais $(14,15$ e $16 \mathrm{~km} / \mathrm{h}$, $p<0,01$ ). Ao mesmo tempo, verificou-se para a FP (gráfico 2), maiores valores de FP nas maiores velocidades $(p<0,01)$. Marcadores articulares de dois voluntários foram danificados durante os testes, de forma que a análise cinemática totalizou $n=11$.

Com os valores de AP e FP foi calculado o percentual de alteração entre os estágios (gráfico 3), sendo evidenciada grande alteração inicial para os valores de AP, a qual diminui a taxa de aumento com o desenvolvimento do teste (a partir de $12 \mathrm{~km} / \mathrm{h}$ ). Houve diferença significante entre primeira transição (8-9km/h) em relação à 10-11, 11-12, 12-13, 1314 e 14-15km/h ( $<<0,05)$, como também da segunda transição (9-10) em relação à $13-14$ e $14-15 \mathrm{~km} / \mathrm{h}(p<0,05)$. De maneira interessante, o comportamento da FP é diferente, apresentando aumentos suaves e não significantes ( $p>0,05$ ) entre todas as transições. Não foram verificadas correlações significantes entre os parâmetros cinemáticos (\% alteração da AP e FP) e parâmetros antropométricos (massa, altura, comprimento da coxa e comprimento da perna) $(p>0,05)$.

As correlações entre as variáveis do experimento foram sintetizadas na tabela 2. Verifica-se que existem correlações fracas de delta-GLI e parâmetros antropométricos (massa, altura, \%gord.) e também em relação ao estado de treinamento (N-TR). Não houve também correlação considerável entre o delta-GLI e o número de estágios realizados. Por outro lado o delta-LAC se correlacionou inversamente de maneira moderada e significante $(p<0,05)$ com a massa e a altura, não havendo correlação com o \%gord. De maneira interessante, houve correlação inversa, moderada e significante entre o delta-LAC e o nível de treinamento dos voluntários $(p<0,05)$, sem haver correlação entre o delta-LAC e o número de estágios analisados. 


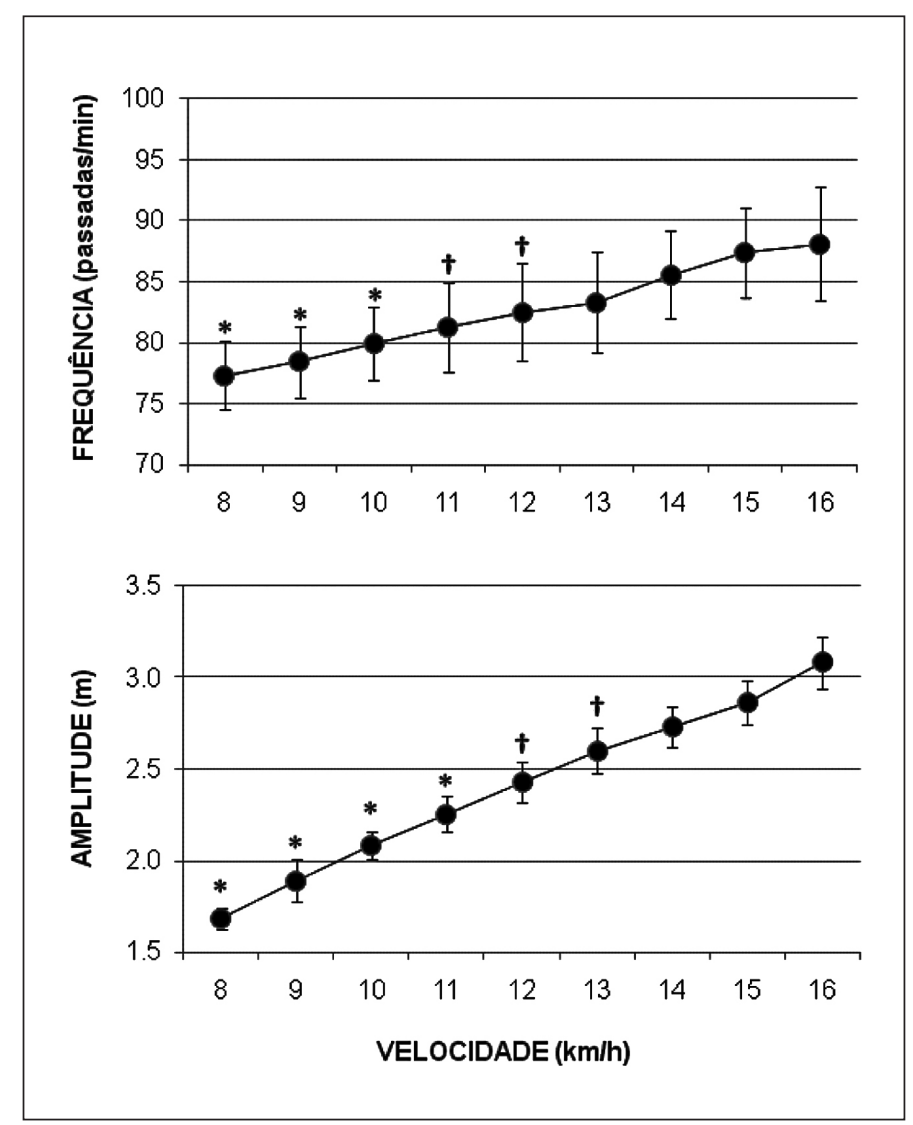

* Diferença significante em relaçăo às velocidades de 14, 15 e 16km/h (one-way Anova) † Diferença significante em relaçăo à velocidade de $16 \mathrm{~km} / \mathrm{h}$ (one-way Anova)

Gráfico 2. Valores médios e desvio padrão da frequência de passadas (acima), e amplitude de passadas (abaixo), para as velocidades utilizadas durante o teste incremental em esteira rolante $(n=11)$.

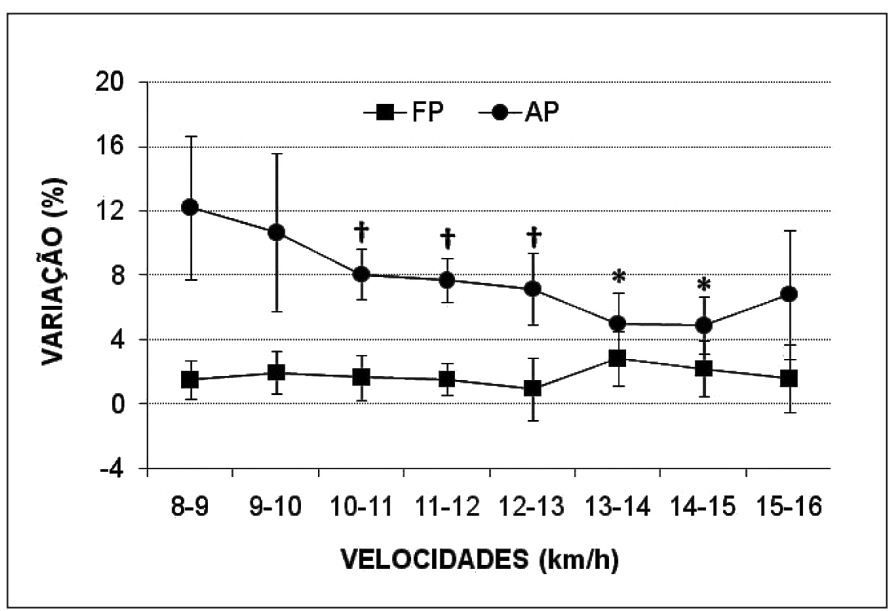

* Diferença significante em relação a 8-9 e 9-10km/h (one-way Anova)

† Diferença significante em relação a $8-9 \mathrm{~km} / \mathrm{h}$ (one-way Anova)

Gráfico 3. Valores médios e desvio padrão da variação (aumento em porcentagem) da amplitude (AP) e frequência de passadas (FP) durante o teste incremental em esteira rolante $(n=11)$.

Tabela 2. Valores de coeficiente de correlação dos valores de delta-GLI e delta- LAC em relação a massa corporal, altura, percentual de gordura, nível de treinamento ( $\mathrm{N}-\mathrm{TR}=$ \%vMAX), e número de estágios realizados durante o teste incremental (EST). * denota significância da correlação $(p<0,05)(n=11)$.

\begin{tabular}{c|c|c|c|c|c}
\hline & Massa & Altura & \%gord & N-TR & EST \\
\hline Delta-GLI & 0,38 & 0,08 & 0,25 & 0,05 & 0,10 \\
\hline Delta-LAC & $-0,43^{*}$ & $-0,66^{*}$ & $-0,2$ & $-0,63^{*}$ & 0,14 \\
\hline
\end{tabular}

\section{DISCUSSÃO}

Avaliações físicas frequentemente utilizam testes de aptidão aeróbia, como o teste incremental, uma vez que índices como o LAn se mostram muito atrativos para a prescrição de treinamentos. O presente estudo apresentou resultados esperados nas concentrações sanguíneas de glicose e lactato, e também aumentos de parâmetros cinemáticos (AP e FP). No entanto, os principais achados são as relações que parâmetros antropométricos apresentam, principalmente, com o lactato sanguíneo. Neste sentido, os resultados do presente estudo oferecem dados normativos do comportamento de pessoas fisicamente ativas durante teste incremental em esteira rolante, além de estimativa sobre como pode ser o comportamento de variáveis fisiológicas ([GLI] e [LAC]) frente à antropometria do indivíduo.

Durante exercício incremental, a necessidade de manutenção de energia é fator decisivo para desempenho da atividade, e um dos mecanismos para a manutenção energética é a utilização da glicose. A [GLI] aumenta quando o exercício é realizado em intensidades altas e também por períodos longos de tempo (Powers, Howley, 2005) e, no caso específico de testes incrementais, existe aumento importante da [GLI], pois a transição entre os sistemas aeróbio/anaeróbio acarreta predominância da utilização do metabolismo anaeróbio a partir da intensidade representativa do $L A n^{(1,13)}$. Tais evidências também foram verificadas no presente estudo, no qual a glicose foi aumentada após o exercício incremental em esteira.

Ao mesmo tempo, outro resultado esperado foi o aumento da [LAC] após o teste incremental em esteira. Diversos autores já descreveram concentrações altas de lactato após testes incrementais, as quais variam entre 8 e $14 \mathrm{mmol} . \mathrm{L}^{-1(14,15)}$. No presente estudo verificou-se valor médio de 9,4 $\pm 2 \mathrm{mmol}^{-L^{-1}}$, que está de acordo com os valores encontrados em estudos anteriores, que utilizam um tempo entre zero e 10 minutos após o exercício para a medição da alteração da [LAC] decorrente da realização do teste ${ }^{(14,15)}$, atingindo seu pico entre três e cinco minutos após o exercício ${ }^{(15)}$, período no qual o presente estudo realizou a coleta da amostra de sangue. O aumento da [LAC] é indicador da mobilização do metabolismo anaeróbio ao longo do exercício(16), e tem relação com a perda da manutenção de desempenho por meio da utilização dos mecanismos da oxidação(15). Sendo assim, o aumento da [LAC] apresenta-se como ferramenta útil de estudos para verificar as formas de utilização das vias metabólicas durante um exercício.

Foi demonstrado que pode haver aumento concomitante da FP e AP durante o exercício incremental, o que possui relação com a necessidade de deslocamento mais rápido com o aumento da velocidade de teste $e^{(9,10)}$. Tais alterações são necessárias para a manutenção de consumo de oxigênio durante o exercício, diminuindo a demanda metabólica, uma vez que a economia de corrida relaciona-se com a otimização dos parâmetros cinemáticos ${ }^{(9)}$. Mesmo assim, verifica-se que ao final do exercício existe grande concentração de metabólitos, pois os membros inferiores realizam movimentos mais rápidos e com maior amplitude, alterando assim o recrutamento muscular ${ }^{(6)}$, e a fadiga se torna inevitável a partir de determinado grau de exigência.

Tal relação pode ser verificada pela diminuição na taxa de aumento da amplitude da passada dos voluntários do presente estudo. Foi encontrado um LAn de 11,1 $\pm 1,4 \mathrm{~km} / \mathrm{h}$, e de maneira interessante, a partir desta intensidade verifica-se menor taxa de aumento para os voluntários do presente estudo. Existe limitação metodológica para a determinação com alta precisão para este parâmetro cinemático, uma vez que o teste é realizado em esteira rolante; no entanto, a padronização da determinação diminui a variabilidade intersujeitos.

O presente estudo investigou também possíveis relações entre fatores antropométricos e as respectivas alterações que parâmetros metabólicos poderiam apresentar. Quanto à [GLI], nenhum parâmetro 
antropométrico demonstrou qualquer relação. Neste sentido, é importante ressaltar que os mecanismos de utilização da glicose sanguínea apresentam-se ligados a descargas de hormônios (adrenalina, glucagon e cortisol) responsáveis pelos processos de glicogenólise durante o exercício para que exista o aumento de sua concentração em momentos necessários ${ }^{(1,16)}$. Este mecanismo envolve a enzima fosforilase, que é liberada quando existe aumento da concentração de $\mathrm{Ca}^{++}$ sarcoplasmático ${ }^{(13)}$, independentemente do volume muscular utilizado ou da técnica de execução do gesto desportivo. Sendo assim, fatores antropométricos parecem não apresentar relação com a liberação deste substrato ao longo de um exercício incremental.

Por outro lado, a [LAC] apresentou-se correlacionada com alguns parâmetros antropométricos, o que remete algumas inferências interessantes. Como evidenciado anteriormente, o lactato sanguíneo possui como uma de suas principais formas de liberação, a utilização do metabolismo glicolítico, devido a intensidades que não podem ser sustentadas pelas vias oxidativas apenas, não sendo atualmente considerado fator indutor de fadiga ${ }^{(15)}$. O presente estudo demonstrou que fatores como a massa e a altura apresentam certa influência, apesar de pequena, em relação à quantidade de lactato sanguíneo que pode ser encontrada após um exercício exaustivo e de curta duração. Estudos anteriores verificaram que a [LAC] pode ser influenciada por diversos fatores, como o tipo de exercício, a quantidade de massa muscular envolvida, a intensidade do exercício entre outras ${ }^{(1,13,16,18)}$. Neste sentido, as correlações encontradas no presente estudo enriquecem o conhecimento quanto a fatores que podem estar relacionados à alterações do lactato, uma vez que voluntários que apresentaram maior massa corporal (em kg) e também maiores estaturas (em cm) foram os voluntários que apresentaram menores aumentos relativos após o teste.

Tais comportamentos das correlações podem ser explicadas pela interpretação de alguns resultados obtidos. A amostra do presente estudo apresentou correlação moderada e significante entre a massa e a altura ( $r=0,60, p=0,02)$, demonstrando que os voluntários mais altos eram, em sua maioria, aqueles com maior massa corporal. Sendo assim, a antropometria desses voluntários, independente do condicionamento, apresentou um padrão. Verifica-se que voluntários altos e com mais massa apresentaram comportamentos diferentes em relação a voluntários mais baixos com menor massa quanto a [LAC]. Com isso, pode-se inferir que quanto mais baixa a pessoa, maior será a concentração final de lactato. Uma das razões para este comportamento pode ser a menor massa muscular envolvida, diminuindo a possibilidade de utilização do lactato como fonte energética para músculos periféricos ${ }^{(16)}$, ou mesmo para os músculos ativos, que para pessoas de maior estatura apresentaram-se mais representativos na composição corporal na população estudada.

\section{REFERÊNCIAS}

1. Denadai BS. Avaliação aeróbia. determinação indireta da resposta do lactato sanguíneo. Rio Claro: Motrix, 2000.

2. Ribeiro JP. Limiares metabólicos e ventilatórios durante o exercício. Aspectos fisiológicos e metodológicos. Arquivos Brasileiros de Cardiologia 1995;64:171-81

3. Bentley DJ, Newell J, Bishop D. Incremental exercise test design and analysis: Implications for performance diagnostics in endurance athletes. Sports Med 2007;37:575-86.

4. Simões HG, Campbell CSG, Kushnick MR, Nakamura A, Katsanos CS, Baldissera V, Moffat RJ. Blood glucose threshold and the metabolic responses to incremental exercise tests with and without prior lactic acidosis induction. Eur J Appl Physiol 2003;89:603-611.

5. Oliveira AS, Caputo F, Gonçalves M, Denadai BS. Heavy-intensity aerobic exercise affects the isokinetic torque and functional but not conventional hamstrings:quadriceps ratios. J Electromyogr Kinesiol. 2008; In press.

6. Hanon C, Thépaut-Mathieu C, Vandewalle H. Determination of muscular fatigue in elite runners. 2005;94:118-25.

7. Bus SA. Ground reaction forces and kinematics in distance running in older-aged men. Med Sci Sports Exerc 2003;35:1167-75
O percentual de gordura corporal relacionou-se com a [LAC], assim poderia se esperar que os voluntários com maior percentual de gordura apresentassem maiores concentrações de lactato devido à menor possibilidade de utilização da massa magra para a remoção deste lactato; entretanto, esta hipótese não foi confirmada. De maneira interessante, o nível de treinamento que pode ser determinado no presente estudo também não se correlacionou com o percentual de gordura, demonstrando que os voluntários com menor percentual de gordura não necessariamente são os que possuem melhor aptidão aeróbia. Provavelmente outros fatores não controlados no presente estudo (força muscular, técnica de corrida, motivação) possam explicar o melhor desempenho de pessoas que não possuem o menor percentual de gordura.

O presente estudo apresentou correlação moderada entre o nível de treinamento $\left(\% \mathrm{VVO}_{2}\right.$ max - no caso do presente estudo a \%vMAX, uma vez que valores de $\mathrm{VO}_{2}$ não foram registrados por meio do ventilômetro) e a [LAC ${ }^{(1)}$. Espera-se que pessoas com melhor condicionamento consigam alcançar maiores velocidades de corrida em um teste máximo, uma vez que a utilização das vias metabólicas é otimizada e lentifica o inevitável efeito dos mecanismos da fadiga muscular. O presente estudo demonstrou que os voluntários mais condicionados não são os que apresentam menores percentuais de gordura, mas apresentam os menores acúmulos de lactato ao final do teste. Uma das explicações seria a maior capacidade do sistema muscular em utilizar esse lactato produzido como fonte de energia durante o próprio exercício(13) uma vez que o tempo destes voluntários em intensidades acima do LAn (intensidade que marca o início do acúmulo desordenado de lactato) é menor. Sendo assim, essa verificação do aumento da [LAC] após o teste incremental pode ser ferramenta adicional para estipular o nível de condicionamento de praticantes recreativos e atletas de corrida.

\section{CONCLUSÕES}

O presente estudo demonstrou que existe aumento de parâmetros cinemáticos (FP e AP) durante o exercício incremental, acompanhado de aumentos das concentrações de glicose e lactato sanguíneo após o teste. No entanto, não existe relação direta entre as alterações nas [GLI] e [LAC], pois cada um destes substratos é controlado por diferentes formas durante o exercício. Além disso, o lactato pode estar mais relacionado com fatores antropométricos, evidenciando que pessoas maiores e com maior massa corporal apresentam menores concentrações, independente do \% de gordura corporal.

Todos os autores declararam não haver qualquer potencial conflito de interesses referente a este artigo.

8. Cavanagh PR, Kram R. Stride length in distance running: velocity, body dimensions, and added mass effects. Med Sci Sports Exerc 1989;21:467-479.

9. Hunter I, Smith GA. Preferred and optimal stride frequency, stiffness and economy: changes with fatigue during a 1-h high-intensity run. Eur J Appl Physiol 2007;100:653-61.

10. Paavolainen L, Nummela A, Rusko H. Neuromuscular characteristics and muscle power as determinants of 5-km running performance. Med Sci Sports Exerc 1999;31:124-30.

11. Silva PB, Fraga CHW, Silva SRD, Cardozo AC, Goncalves M. EMG and kinematic analysis of an incremental running test. In: Proceedings International Symposium of Biomechanics in Sport, 2007.

12. Jackson AS, Pollock ML. Generalized equations for predicting body density of men. Br J Nutr 1978;40:497-504

13. Powers SK, Howley ET. Fisiologia do exercício. Teoria e aplicação ao condicionamento e ao desempenho. São Paulo: Manole, 2005

14. Denadai BS. Índices fisiológicos de avaliação aeróbia. conceitos e aplicações. 1. ed. Ribeirão Preto: Bsd, 1995.

15. Gladden LB. Lactate metabolism: a new paradigm for the third millenium. J Physiol 2004;558:5-30.

16. Oliveira AS, Greco CC, Pereira MP, Figueira TR, Ruas VDA, Gonçalves M, Denadai BS. Acute responses during a high-resistance training session. J Strength Cond Res 2009;23:579-86. 